
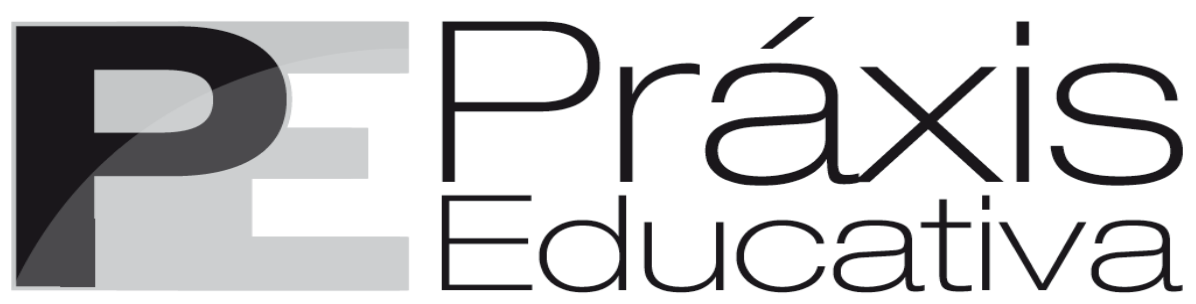

ISSN 1809-4031

eISSN 1809-4309

https://doi.org/10.5212/PraxEduc.v.16.18097.072

\title{
Beneficios de los MOOC en estudiantes universitarios durante la emergencia académica por la Covid-19
}

\section{MOOC's benefits for higher education students during the academic emergency due to Covid-19}

\section{Benefícios dos MOOC em estudantes universitários durante a emergência acadêmica devido à Covid-19}

\author{
Salvador Ponce Ceballos* \\ iD https://orcid.org/0000-0003-0454-9853 \\ Paul Yitzen Ruelas Mexía* \\ (iD https:/ / orcid.org/0000-0002-2235-0162
}

Resumen: La contingencia académica por la covid-19 provocó un aceleramiento en la suma de iniciativas de educación en línea en el ámbito de la educación superior. Entre ellas, los MOOC (Massive Open Online Course). El presente artículo documenta un ejercicio de investigación sobre la experiencia de una universidad pública mexicana que, durante la pandemia, incorporó dentro de su propuesta formativa la posibilidad de que sus estudiantes cursaran MOOC. El estudio de tipo cualitativo, basado en el análisis de contenido, tuvo como objetivo identificar, desde la óptica de los estudiantes, los beneficios de haber participado en este tipo de experiencias formativas. Los resultados indican un conjunto de beneficios integrados en seis categorías: desarrollo académico, desarrollo profesional, desarrollo personal y motivación, flexibilidad, internacionalización y continuidad académica. La conclusión se orienta a confirmar la existencia de beneficios y que dentro de ellos hay particularidades asociadas al escenario de contingencia académica.

Palabras clave: MOOC. Covid-19. Educación superior.

Abstract: The academic contingency due to covid-19 caused an acceleration in the sum of online education initiatives in higher education, including MOOC (Massive Open Online Course). This article documents a research exercise on the experience of a Mexican public university, which, during the pandemic, incorporated

* Doctor en Educación por la Universidad Abierta de Tlaxcala. Profesor-investigador de la Universidad Autónoma de Baja California. E-mail: ponce@uabc.edu.mx

${ }^{* *}$ Licenciado en Docencia de la Lengua y Literatura por la Universidad Autónoma de Baja California. Analista de la Universidad Autónoma de Baja California. E-mail: ruelasp@uabc.edu.mx

Práxis Educativa, Ponta Grossa, v. 16, e2118097, p. 1-24, 2021

Disponível em: <https://revistas2.uepg.br/index.php/praxiseducativa> 
into its teaching proposal the possibility of its students taking some MOOC. The qualitative study, based on content analysis, aimed to identify, from the students' point of view, the benefits of participating in this type of learning experiences. The results indicate a set of benefits integrated into six categories: academic development, professional development, personal development and motivation, flexibility, internationalization and academic continuity. The conclusion is aimed at confirming the existence of benefits and that within them there are particularities associated with the academic contingency scenario.

Keywords: MOOC. Covid-19. Higher education.

Resumo: A contingência acadêmica decorrente da covid-19, ocasionou uma aceleração na soma das iniciativas de educação online na área de ensino superior, inclusive MOOC (Massive Open Online Course, por sua sigla em inglês). Este artigo documenta um exercício de pesquisa sobre a experiência de uma universidade pública mexicana que, durante a pandemia, incorporou em sua proposta de formação a possibilidade de seus alunos cursarem alguns MOOC. O estudo qualitativo, baseado na análise de conteúdo, objetivou identificar, na visão dos alunos, os benefícios de terem participado desse tipo de experiência de formação. Os resultados indicam um conjunto de benefícios integrados em seis categorias: desenvolvimento acadêmico, desenvolvimento profissional, desenvolvimento e motivação pessoal, flexibilidade, internacionalização e continuidade acadêmica. A conclusão visa confirmar a existência de benefícios e que neles existem particularidades associadas ao cenário de contingência acadêmica.

Palavras-chave: MOOC. Covid-19. Educação Superior.

\section{Introducción}

La educación en línea ha tenido un aumento importante en los últimos años (ALLEN; SEAMAN, 2016), dando mayor posibilidad de estudiar a quienes no pueden ingresar a la oferta de programas educativos presenciales (DZIUBAN; GRAHAM; MOSKAL; NORBERG; SICILIA, 2018; OEI, 2019). Una de las estrategias más recientes en este tipo de modalidades en línea son los MOOC (Massive Open Online Course). Estos han cobrado alta importancia en el ámbito universitario, tanto en las instituciones públicas como en las privadas, ya sea desde la óptica de Recursos Educativos Abiertos (REA) o como cursos (STRACKE; DOWNES; CONOLE; BURGOS; NASCIMBENI, 2019). En cualquiera de las dos, nos estamos refiriendo a las tecnologías asociadas al aprendizaje, las cuales, desde su nacimiento, han demostrado aportes significativos.

La incorporación de la tecnología en la educación, para la trasformación de las instituciones, se ha impulsado desde hace muchos años (KOPP; GRÖBLINGER; ADAMS, 2019). Esto ha provocado el surgimiento y desarrollo de modalidades y estrategias para la formación de vanguardia (NAGASHIMA, 2014).

Sin embargo, la realidad es que la situación emergente a causa de la enfermedad denominada covid-19, originada en diciembre de 2019 (BRYSON; 2021), promovió que se llevara a cabo una repentina y obligada transformación digital (RIBEIRO, 2020; BROWN, 2020), la cual demostró la importancia de la tecnología en el ámbito educativo; en particular, la de la educación en línea.

Ante esta situación, continuar con los servicios educativos implicó que se probaran estrategias a las que no se les había dado gran aceptación (AKURATIYA; MEDDAGE, 2020), como el caso de los MOOC, los cuales, aunque se debate sobre sus beneficios y desventajas (BORDEN, 2014), en algunas instituciones ayudaron a mitigar la necesidad emergente de diseños en línea de calidad.

Antes de la pandemia, algunas instituciones ya exploraban opciones de incorporación en sus currículums de este tipo de cursos (FIRMIN; SCHIORRING; WHITMER; WILLETT; COLLINS;

Práxis Educativa, Ponta Grossa, v. 16, e2118097, p. 1-24, 2021 Disponível em: <https://revistas2.uepg.br/index.php/praxiseducativa> 
SUJITPARAPITAYA, 2014), tanto en el plano del fortalecimiento de los distintos aprendizajes de los estudiantes, como dentro de la formalidad de sus currículos en cursos obligatorios u optativos. Asimismo, algunas han desarrollado sus propias propuestas y otras han optado por las plataformas de instituciones u organizaciones externas, como Coursera, Udacity, edX, entre otras. Estas, por cierto, han dado un salto cuántico en menos de 10 años desde su aparición.

Con base en lo anterior, es importante revisar cómo este tipo de estrategias se desarrollaron ante un escenario de contingencia académica (PURKAYASTHA; SINHA, 2021), y con ello valorar dichas estrategias como mecanismos de fortalecimiento en la formación de universitarios en tiempos de contingencia.

Un ejemplo de lo mencionado fue lo sucedido en la universidad mexicana en donde se llevó a cabo el presente estudio, la cual, a principios de marzo de 2020, trasladó las diversas actividades universitarias a la modalidad a distancia, atendiendo las indicaciones de la Secretaría de Educación Pública sobre cancelar las actividades presenciales, debido a la contingencia sanitaria por la pandemia de la covid-19. Lo anterior, lo llevó a cabo mediante la implementación del plan de continuidad académica con el que cuenta la universidad. Esto implicó que las tareas académicas, organizacionales y tecnológicas se llevaran a cabo a través de un esquema a distancia, lo cual incluye las clases para los estudiantes de la institución.

Ante este escenario, la institución incorporó dentro de su propuesta formativa la posibilidad de que los alumnos, de forma voluntaria, cursaran algunos MOOC, a partir de un convenio con la plataforma Coursera. Esto, con la finalidad de atenuar las consecuencias surgidas por la situación de contingencia y poder finalizar el semestre escolar.

De esta manera, el presente artículo documenta un ejercicio de investigación sobre la experiencia de los estudiantes de dicha universidad al cursar un MOOC durante la pandemia. El estudio tuvo como objetivo identificar, desde la óptica de los estudiantes de licenciatura, los beneficios de haber participado en este tipo de experiencias formativas.

El trabajo cuenta con un apartado teórico que busca ilustrar las generalidades de los MOOC, así como los beneficios en la formación de estudiantes universitarios, además de un espacio donde se aborda el contexto de la contingencia sanitaria por la covid-19. Posteriormente, se documenta la metodología de investigación empleada, además de los resultados y conclusiones.

\section{Generalidades de los MOOC}

Los Cursos Masivos en Línea y Abiertos es la forma de referirse, en español, a los Massive Online Open Course (MOOC, por sus siglas en inglés) (RUIZ, 2015). Dicho acrónimo fue sugerido por Dave Cornier y Brian Alexander en 2008 (GRAINGER, 2013). Como su nombre indica, las principales características de los MOOC consisten en que son cursos que se desarrollan en línea, que son abiertos y se llevan a cabo masivamente permitiendo la participación de cientos de estudiantes al mismo tiempo (CASTAÑO; CABERO, 2013).

Son considerados como una modalidad de aprendizaje que ofrece acceso gratuito a las propiedades intelectuales de diversas universidades, incluyendo el contacto con sus estudiantes y profesores de renombre (RIEBER; 2016). Este tipo de cursos están fundamentados en los recursos

Práxis Educativa, Ponta Grossa, v. 16, e2118097, p. 1-24, 2021 
educativos abiertos (REA) propuestos por la Organización de las Naciones Unidas para para la Educación, la Ciencia y la Cultura (UNESCO, por sus siglas en inglés) (RUIZ, 2015).

El primer MOOC fue desarrollado en la Universidad de Manitoba, por parte de George Siemens y Stephen Downes (MACKNESS; WAITE; ROBERTS; LOVEGROVE, 2013). Dicho curso se llevó a cabo en 2008, y aunque es considerado como el primer MOOC oficial, ya existían cursos similares, pero sin aplicar exactamente el modelo de los MOOC (DOWNES, 2012).

A partir de la Declaración de París en 2012, sobre los REA por parte de la UNESCO, donde se recomendó que los países miembros promovieran y utilizaran los recursos educativos abiertos con el fin de ampliar el acceso a la educación (RUIZ, 2015), las universidades han lanzado diversos MOOC y empleado diferentes plataformas y redes tales como Coursera, Future Learn, MiríadaX, Canvas y Creative Commons (MELLATI; KHADEMI, 2018).

En cuanto a los tipos de MOOC, existen dos principales, los cuales se clasifican dependiendo de sus enfoques teóricos: cMOOC (basados en el conectivismo) y xMOOC (fundamentados en el conductismo) (RIEBER, 2016). Respecto a sus características, los cMOOC son flexibles, sin una estructura definida y con acercamientos no lineales, mientras que los xMOOC son más estructurados y adoptan un acercamiento lineal (BOZKURT; KEEFER, 2017).

El primer curso considerado como cMOOC fue justamente el primer MOOC de la historia, denominado Conectivismo y conocimiento conectivo, creado por Stephen Downes y George Siemens. Este cMOOC se caracterizó por no centrarse en los contenidos, sino que hizo énfasis en formar redes entre los participantes para fomentar el intercambio de recursos, así como las contribuciones dentro de las mismas redes. A la pedagogía empleada en este tipo de MOOC se le conoce como conectivista (STRACKE; DOWNES; CONOLE; BURGOS; NASCIMBENI, 2019).

Por otra parte, en 2011, surgieron los xMOOC. Su diseño enfatiza una instrucción más tradicionalista, pues es dirigida por instructores y tiene un enfoque en los contenidos. Además, buscan proporcionarse de manera masiva a los participantes (STRACKE; DOWNES; CONOLE; BURGOS; NASCIMBENI, 2019). Se considera que el primer xMOOC fue el curso llamado Inteligencia Artificial, de Norvig y Thrun, el cual contó con la participación de más de 150,000 personas (STRACKE; DOWNES; CONOLE; BURGOS; NASCIMBENI, 2019).

Existe, además, un tipo de MOOC que es una especie de híbrido entre los tipos anteriores. Éste se llama tMOOC y se centra en la tarea, además de enfocarse en el desarrollo de competencias para desempeñarse en un tipo determinado de trabajo. Aquí, el estudiante debe realizar satisfactoriamente y cumplir con tareas obligatorias durante todo el curso (RUÍZ, 2015).

Sin embargo, a pesar de las diferencias entre los tipos de MOOC, Conole (2015) establece que todo MOOC debe cumplir con las siguientes 12 dimensiones. Deben ser: (1) abiertos, (2) masivos y (3) diversos. Asimismo, deben considerar la (4) multimedia, (5) comunicación, (6) colaboración, (7) reflexión, (8) ruta del aprendizaje, (9) aseguramiento de la calidad, (10) certificación, (11) aprendizaje formal y (12) autonomía. 


\section{Beneficios e impacto de los MOOC en el ámbito educativo}

A partir de la década pasada, se presentó un crecimiento del uso del Internet, así como de las redes sociales, teniendo como consecuencia un exponencial uso de la tecnología en la vida diaria y los diversos aspectos que en ella intervienen, como es la educación (CRIPPS, 2014). Esto implica que la tecnología ha permitido incrementar las posibilidades de estrategias de enseñanza y de las herramientas a utilizar en los procesos educativos, por lo que identificar los beneficios de una estrategia como los MOOC aporta una valoración de su utilidad. En este apartado, se presenta un análisis de literatura especializada concentrada específicamente en los beneficios e impacto de los MOOC en el ámbito educativo.

De acuerdo con Loizzo y Ertmer (2016), uno de los beneficios de este tipo de cursos consiste en que implementan diversas herramientas digitales, las cuales, a través del uso que los alumnos tienen que hacer de ellas, les permiten desarrollar un aprendizaje. De igual forma, parte de su impacto se debe a que los cursos cuentan con fechas de comienzo y de finalización, así como con mecanismos de evaluación, asemejándose a una clase (CASTAÑO; CABERO, 2013). De esta manera, también es posible que el estudiante reciba una retroalimentación y su progreso pueda ser monitoreado y evaluado (ALHAZZANI, 2020).

Otras ventajas consideradas por Castaño y Cabero (2013), así como Purkayastha y Sinha (2021), consisten en que sean online, de uso gratuito y abiertos. Estas mismas características, es decir, que sean abiertos y en modalidad online, contribuyen a otro impacto positivo, como es el hecho de poner a disposición de los participantes, de manera libre, diversos contenidos y materiales con los que pueden interactuar y de esta manera convertirlos en el centro del aprendizaje (DURALL; GROS; MAINA; JOHNSON; ADAMS, 2012). Esto, a través de materiales tales como presentaciones en video, cuestionarios cortos y exámenes disponibles en línea, tal y como se maneja en los xMOOC (SIEMENS, 2012), los cuales suelen ser buenos materiales que facilitan el entendimiento de los contenidos, ya que permiten que los participantes realicen una repetición de los mismos en caso de ser necesario (CRIPPS, 2014).

Asimismo, la justificación de que dichos materiales contribuirán al desarrollo de un aprendizaje consiste en que estos, así como los contenidos, son seleccionados por los académicos y profesionales que forman parte de las universidades y/o instituciones que ofertan los MOOC (CABERO; LLORENTE; VÁZQUEZ, 2014), convirtiéndose, de esta manera, en contenidos de calidad, al contar con el respaldo de profesores de alto nivel (AMERICA LEARNING MEDIA, 2002), y al estar diseñados con la intención de mejorar conceptos y conocimientos (ALHAZZANI, 2020), además de facilitar el proceso de aprendizaje a través de la oferta de dichos materiales y compartir información (AL-RAHMI; ALDRAIWEESH; YAHAYA; BIN; ZEKI, 2019).

Existen diversos estudios y autores que tratan el tema de los beneficios de los MOOC no solo en el ámbito educativo, sino también para el personal y profesional. Por ejemplo: Padilla, Rocha y Rodríguez (2017) mencionan, de forma puntual, que los MOOC resultan benéficos porque los estudiantes que se integran a uno por interés en un tema en específico, pueden abandonar el curso al aprender lo que deseaban sin necesidad de avanzar más en el curso. También, porque los participantes pueden definir la importancia que los cursos tienen para ellos, sin preocuparse por una evaluación demasiado formal, además de que los MOOC representan una oportunidad educativa para cualquier persona que desee tomarla.

Práxis Educativa, Ponta Grossa, v. 16, e2118097, p. 1-24, 2021

Disponível em: <https://revistas2.uepg.br/index.php/praxiseducativa $>$ 
De la misma manera, Díaz, Baena y Baena (2017) consideran que la pertinencia de los MOOC radica en que, al ser online, son una opción educativa para quienes no cuentan con el tiempo o disponibilidad para cursar una modalidad presencial; también, en el ámbito profesional, porque representan una opción más para profesionistas que quieren capacitarse y actualizarse en diversos temas o habilidades que requieren en su área de trabajo; del mismo modo, porque amplían las oportunidades de acceso a información de confianza y que está actualizada, con lo que se pueden satisfacer necesidades específicas. Agregan, también, que resultan adecuados porque el alumno funge como un creador de su propio conocimiento, donde el docente o instructor actúa más como un facilitador.

Por otra parte, Laborde y Ortega (2016) mencionan que los MOOC presentan los siguientes siete beneficios principales: (1) acortamiento de la brecha del conocimiento; (2) ahorro de tiempo y comodidad, debido a que se puede acceder a ellos independientemente de la localización siempre y cuando se cuente con internet; (3) aterrizaje de la teoría a la realidad; (4) incrementan la red de contactos, ya que permiten la interacción y conexión entre personas de diversos lugares del mundo; (5) flexibilidad, porque los participantes pueden llevar a cabo las actividades de acuerdo con sus tiempos, acomodándose a lo que más les convenga o las posibilidades que tengan; (6) son aptos para todos, pues no exigen que los participantes cuenten con conocimientos previos, además de que los contenidos son fáciles de entender, haciéndolos más accesibles; (7) y conocimiento dinámico que está en una evolución constante, el cual se complementa con la participación de los otros integrantes del curso, quienes aportan sus propias experiencias y comentarios.

Zambrano (2017) concuerda con varios de estos beneficios mencionados anteriormente, al indicar que los MOOC permiten: flexibilidad de estudio en cuanto a tiempo y lugar, adquisición de nuevos conocimientos, interacción entre personas del mundo entero, facilidad de acceso a todo tipo de estudiantes, así como acceso a un aprendizaje de calidad. Esta última característica, debido a que los cursos son desarrollados por las mejores universidades.

Con base en lo anterior, es que este tipo de cursos se visualizan como un medio que impulsa la internacionalización (RIEBER, 2016).

Asimismo, se considera que, en cuanto al aspecto académico, la dinámica y diseño de los MOOC contribuyen a incrementar la calidad de la docencia, facilitar el acceso a la formación y contribuir a la innovación en el aprendizaje, puesto que permiten diversificar las metodologías docentes (GÓMEZ, 2014). Pero, también pueden ofrecer otro tipo de beneficios potenciales, tales como ayudar al logro de una mayor relación entre la educación superior y el ámbito laboral, mejorar la presencia de las universidades en un marco internacional y convertirse en medios para difundir conocimientos mediante una colaboración global, además de favorecer las oportunidades de formación para cualquier persona, incluso profesionales (GÓMEZ, 2014).

Al igual, los MOOC son considerados como un instrumento sólido que permite la transformación y mejora del proceso de enseñanza-aprendizaje, proporcionándole una mayor calidad por el hecho de tener un enfoque en el aprendizaje entre pares y el fomento de creación de comunidades en línea, lo cual permite la colaboración y comunicación entre los alumnos (STRACKE; DOWNES; CONOLE; BURGOS; NASCIMBENI, 2019). Esto último, logrado gracias también a que los MOOC permiten una interacción a gran escala de cientos de estudiantes, por el hecho de ser abiertos y no contar con criterios de admisión (CASTAÑO; CABERO, 2013), lo que permite que todo tipo de persona y estudiantes puedan unirse.

Práxis Educativa, Ponta Grossa, v. 16, e2118097, p. 1-24, 2021 Disponível em: <https://revistas2.uepg.br/index.php/praxiseducativa> 
Por otro lado, se han realizado estudios, como el de Cripps (2014), donde se identificaron los beneficios para quienes han tomado un MOOC. Los resultados arrojaron que los participantes visualizan como beneficio la amplia variedad existente en cuanto a las temáticas de los MOOC, pues esto les brinda una oportunidad de incrementar sus aprendizajes y conocimientos; asimismo, comentaron que eran una buena alternativa para personas interesadas en hacer algún estudio de intercambio porque les permite estudiar otras temáticas que les podrían ser útiles.

También se encontró como un aspecto positivo la oportunidad de interactuar con personas del extranjero e incluso con personas de diferentes edades, lo cual enriquece la experiencia (CRIPPS, 2014). Esto último no ha sido comentado únicamente por parte de los participantes de los MOOC, sino que también ha sido expresado en la teoría, como es el caso de Frías, Arquero y Del Barrio-García (2020) quienes mencionaron que uno de los beneficios de los MOOC consiste en la oportunidad de interacción, creación y compartición de conocimientos entre los participantes. De la misma manera, Loizzo y Ertmer (2016) indican que estos cursos ofrecen la oportunidad de experimentar otro tipo de perspectivas de aprendizaje, al permitir la interacción de miles de estudiantes de diversos lugares, características y culturas que poseen interés en un tema en común. Temas que pueden ir desde las humanidades, medicina, biología, ciencias sociales, matemáticas, negocios, informática, entre otros (ALHAZZANI, 2020).

En la opinión expresada por los participantes del estudio de Cripps (2014) también se identificó un punto interesante a tomar en cuenta: se mencionó que los MOOC, de manera indirecta, pueden servir para practicar un idioma, pues los participantes tienen la opción de ingresar a un curso que esté en una lengua diferente a la suya, con la opción (ofrecida por algunas plataformas) de ciertos materiales con subtítulos, lo cual les facilita el entendimiento de los contenidos a pesar de estar en otro idioma.

Los participantes también proporcionaron algunos puntos adicionales al describir a los MOOC como simples, fáciles, divertidos y con herramientas efectivas (CRIPPS, 2014).

Otros estudios, como el de Mellati y Khademi (2018) concluyeron que la integración de los MOOC en el ámbito educativo ha contribuido a la mejora del rendimiento académico. Dicha conclusión se obtuvo al identificar que los participantes que trabajaron en un contexto educativo basado en MOOC superaron académicamente a quienes se desempeñaron en modalidades más tradicionales como las presenciales o de cara a cara. Por un lado, los autores consideran que esto se debió al hecho de trabajar en un modo innovador de aprendizaje. Por otra parte, desde la perspectiva de los estudiantes y docentes involucrados en el estudio, mencionaron que esto se logró gracias a diversos factores como tener mayor control sobre los materiales utilizados y la forma en que estos les eran presentados; que pudieron adquirir nuevas visiones de aprendizaje por compartir con diferentes culturas; que pudieron expresarse con más confianza al no tener que hacer contacto visual directo con los demás participantes, por lo que el estrés era menor; que existió disponibilidad de los materiales y contenidos antes y después del curso; y que tanto los comportamientos como estrategias de enseñanza eran nuevas y promovían la colaboración y ayuda (MELLATI; KHADEMI, 2018)

Otro de los aspectos positivos de los MOOC es la flexibilidad que estos aportan, debido a que los participantes pueden estudiar a su propio ritmo y sin una presión de tiempo u horarios definidos (CRIPPS, 2014); también, el hecho de que los participantes puedan formarse y tomar cursos de instituciones externas a sus propias instituciones de estudio (CRIPPS, 2014; DURALL; GROS; MAINA; JOHNSON; ADAMS, 2012); además, que promueven el desarrollo de habilidades y capacidades, tales como la creación y generación de conocimiento, creatividad, autonomía y

Práxis Educativa, Ponta Grossa, v. 16, e2118097, p. 1-24, 2021 Disponível em: <https://revistas2.uepg.br/index.php/praxiseducativa> 
Beneficios de los MOOC en estudiantes universitarios durante la emergencia académica por la covid-19

aprendizaje a través de las relaciones sociales (SIEMENS, 2012); al igual, que permitan la adquisición de créditos (FRÍAS; ARQUERO; DEL BARRIO-GARCÍA, 2020; CONOLE, 2015) o de constancias y certificados de terminación (LOIZZO; ERTMER, 2016).

Todo lo anterior ha desembocado en otros beneficios indirectos para los estudiantes, como el hecho de que desarrollen motivación para continuar cursando MOOC o para finalizarlos (FRÍAS; ARQUERO; DEL BARRIO-GARCÍA, 2020; ALHAZZANI, 2020), pues ellos mismos identifican los impactos benéficos que estos tienen, además de obtenerlos de manera gratuita, debido a que no se realiza un cobro por ellos (CRIPPS, 2014; ALHAZZANI, 2020) sumado a que la interacción del estudio en grupo también fomenta su motivación (MELLATI; KHADEMI, 2018).

Asimismo, retomando algunos puntos revisados anteriormente, resulta necesario indicar que los cursos MOOC son beneficiosos para el proceso de enseñanza-aprendizaje, ya que su diseño tiene la finalidad de ayudar a difundir nuevos conceptos de forma rápida y eficaz (ALHAZZANI, 2020), además de que la prioridad principal de los mismos es mejorar la calidad del aprendizaje de los participantes que en ellos intervienen (MAZOUÉ, 2013). De esta manera, el diseño de los MOOC ofrece una posibilidad de reemplazar ciertas prácticas de la enseñanza por otras formas de aprendizaje que sean más efectivas y eficientes (MAZOUÉ, 2013), tomando en consideración que el enfoque se centra en ideas clave, el dominio de conceptos y la búsqueda de que los nuevos conocimientos que se busca adquirir sean consistentes (ALHAZZANI, 2020).

Por lo tanto, en términos de generación de conocimiento y como proveedores de información, los MOOC resultan adecuados como actividades de aprendizaje, considerando también que aprovechan la tecnología (AL-RAHMI; ALDRAIWEESH; YAHAYA; BIN; ZEKI, 2019). Por estos mismos motivos, las características de los MOOC se vuelven útiles en el contexto educativo, pero sobre todo en el de educación a distancia, logrando persuadir a estudiantes autodidactas o diseñadores instruccionales a que integren este tipo de tecnologías en sus prácticas de enseñanza, pues resultan eficientes y persiguen los propósitos de un marco pedagógico (MELLATI; KHADEMI, 2018).

Así, Downes (2012) considera que los MOOC pueden empoderar a las personas para que desarrollen y creen su propio aprendizaje, su propia educación. Es decir, que los MOOC permiten que los participantes persigan un aprendizaje de forma autodidacta (AMERICA LEARNING MEDIA, 2002). Esto puede ser logrado mediante la motivación causada por el estudio de algo que les cause un interés personal y que además les proporcione otro tipo de herramientas, como conocimientos útiles para el momento de buscar trabajo, incrementar su currículum al estudiar un MOOC diseñado por una escuela de prestigio que puede ser extranjera o simplemente aprender algo que no aprendieron en la escuela (CRIPPS, 2014).

De manera resumida, podemos asegurar que los beneficios de los MOOC consisten justamente en las características que estos poseen, las cuales son mencionadas en las dimensiones presentadas por Conole (2015): que son abiertos, masivos, utilizan multimedia, fomentan la colaboración y la reflexión; y que presentan un camino para el aprendizaje, mientras aseguran la calidad, además de permitir el logro de una certificación y aprendizaje formal, pero al mismo tiempo con autonomía.

Podemos finalizar este apartado, retomando las ideas de algunos autores, como las de Loizzo y Ertmer (2016), quienes establecen que los MOOC representan una manera de demostrar la cultura cambiante actual, permitiendo oportunidades educativas abiertas para todos, independientemente de sus antecedentes, su locación, profesión, estatus financiero, etcétera. Y, por otro lado, tener en mente

Práxis Educativa, Ponta Grossa, v. 16, e2118097, p. 1-24, 2021 Disponível em: <https://revistas2.uepg.br/index.php/praxiseducativa> 
que los beneficios de los MOOC no consisten únicamente en que sean más convenientes, gratuitos o buenos, sino porque también pueden ser eficaces para contribuir a que los estudiantes desarrollen un mejor aprendizaje (MAZOUÉ, 2013).

\section{La emergencia académica por la covid-19}

La aparición de la pandemia trajo consigo una situación de riesgo para toda la población, por lo que, a partir de marzo de 2020, más de 200 países llevaron a cabo el cierre de las instalaciones de las instituciones educativas (UNICEF, 2021), con la intención de evitar una propagación más rápida del virus y disminuir el impacto de la pandemia (ONYEMA; et al., 2020; CHRISTAKIS; CHRISTAKIS, 2020).

Por este motivo, los sistemas educativos de todo el mundo se vieron obligados a buscar e implementar medidas que contribuyeran a enfrentar las necesidades del momento. Una de las principales fue concluir el ciclo escolar de forma no presencial. Así, la mayoría de los países decidieron apoyarse en la educación a distancia (BROWN, 2020), surgiendo así la Enseñanza Remota de Emergencia (Emergency Remote Teaching, en inglés), que se define como aquella que se trasladó, en poco tiempo, de una enseñanza presencial a una a distancia y que se limita a atender de forma emergente lo que los profesores e instituciones pueden hacer para dar continuidad a las clases (HODGES; MOORE; LOCKEE; TRUST; BOND, 2020). Esta tiene sus antecedentes en la ampliamente estudiada "educación en situación de emergencia" (BURDE; KAPIT; WAHL; GUVEN; SKARPETEIG, 2017).

La educación a distancia implementada durante la pandemia ha enfrentado diversos retos. En principio, ofrecer contenidos y aprendizajes de calidad (UNESCO/IESALC, 2020), apoyados en docentes con el perfil adecuado para este tipo de escenarios (CARMONA; FLORES, 2021), así como la capacidad de los alumnos para el aprendizaje a distancia (HUMAN RIGHTS WATCH, 2021). De igual forma, falta de equipamiento y conectividad (ONYEMA; et al., 2020), tanto para ofrecer clases en línea como para todo tipo de procesos institucionales.

Aunado a lo anterior, las instituciones educativas demostraron una carencia en cuanto a estar preparados con las planeaciones necesarias para enfrentarse a situaciones tales como la de una contingencia sanitaria, por lo que tuvieron que improvisar conforme a la marcha (PARDO; COBO, 2020).

Además de las profundas desigualdades en el ámbito educativo (HUMAN RIGHTS WATCH, 2021; RUSSO; MAGNAN; SOARES, 2020), social, y económico (VALENTI, 2020) mostradas por la pandemia, también se acentuaron problemáticas que, si bien estaban en el discurso educativo, no habían tenido la atención prioritaria, tales como: los problemas emocionales de alumnos y docentes (HUGHES, 2020; DRANE; VERNON; O'SHEA, 2020), la importancia de poner especial interés en los alumnos con discapacidad (COMISIÓN ECONÓMICA PARA AMÉRICA LATINA Y EL CARIBE, 2021) la participación de los padres en la escuela y la formación de sus hijos (NÄSLUNDHADLEY; BIEHL, 2020) y la brecha digital de estudiantes, escuelas y hogares en cuanto equipamiento, conectividad y aprendizaje en línea (HUGHES, 2020), por mencionar algunas.

Práxis Educativa, Ponta Grossa, v. 16, e2118097, p. 1-24, 2021 
Beneficios de los MOOC en estudiantes universitarios durante la emergencia académica por la covid-19

\section{Método}

El presente trabajo corresponde a un estudio de caso, bajo un abordaje cualitativo de alcance exploratorio (MERRIAM; TISDELL, 2015) apoyado en el análisis de contenido, con el objetivo de identificar, desde la opinión de los estudiantes de licenciatura de una universidad pública mexicana, los beneficios de haber cursado un MOOC durante la contingencia académica a causa de la covid-19. En este sentido, dos preguntas guiaron el estudio: ¿Qué beneficios manifestaron los estudiantes? y ¿cuáles de estos beneficios son propios de la emergencia académica?

La dimensión de la evaluación considerada en este trabajo se sitúa desde los beneficios, de acuerdo con el modelo de DeLone y McLean (2003). Dicho modelo es uno de los más utilizados sobre el éxito de los sistemas informáticos (DIRIBA; KANNAN; CHAI, 2021). Inclusive, ha sido adaptado para la evaluación de MOOC, al no existir un modelo propio aceptado en la comunidad académica para este tipo de desarrollos (ALBELBISI, 2020). El modelo se integra por las siguientes dimensiones: calidad de los sistemas, calidad de la información, calidad del servicio, satisfacción de usuario, uso y beneficios.

Se adoptó la dimensión de beneficios debido a las siguientes consideraciones: 1. La importancia de identificar si este tipo de iniciativas realiza aportaciones en la formación de los estudiantes; 2. Identificar la aportación de este tipo de iniciativas en un proceso de contingencia académica; y 3. Estudios previos indican que la utilidad, desde la óptica de los usuarios, está relacionada con la calidad de los servicios tecnológicos (CALISIR; GUMUSSOY; BAYRAKTAROGLU; KARAALI, 2014) y en particular con la de los MOOC (YANG; SHAO; LIU; LIU, 2017).

Respecto a las cuestiones éticas, para el desarrollo de la investigación se trabajó de la siguiente manera: se solicitó el consentimiento a la institución para el uso de la información de los participantes; los participantes contestaron la información solicitada de forma libre y voluntaria; se eliminó toda identificación de datos personales e institucionales; se trabajó con una metodología de investigación rigurosa y respaldada teórica y empíricamente; los resultados fueron procesados sin alteraciones o sesgos.

\section{Contexto}

El estudio se realizó en una universidad pública mexicana que, a partir del mes de marzo de 2020, debido a la emergencia académica por la covid-19, implementó su plan de continuidad académica y trasladó las clases a una modalidad en línea, con el fin de no interrumpir las actividades académicas y al mismo tiempo apoyar a evitar la propagación del virus de la covid-19 al interrumpir actividades presenciales.

La institución ofreció a sus alumnos de licenciatura la posibilidad de cursar, además de su carga académica y de manera voluntaria, cursos MOOC gracias a una alianza con la plataforma Coursera. En total, participaron 4,700 estudiantes, distribuidos en 31 cursos, los cuales fueron gratuitos y tuvieron valor curricular.

Práxis Educativa, Ponta Grossa, v. 16, e2118097, p. 1-24, 2021 


\section{Instrumento}

El ejercicio se apoyó en el instrumento oficial que utiliza la plataforma Coursera para evaluar sus cursos, con base en la opinión de los usuarios; se trata de una pregunta abierta que invita a los usuarios a escribir su retroalimentación sobre el MOOC cursado. Las respuestas a esta pregunta fueron el insumo para el presente ejercicio investigativo.

\section{Participantes}

Participaron 102 estudiantes de licenciatura, de una universidad pública de México, quienes cursaron algún MOOC durante el periodo de mayo a noviembre de 2020, como actividad extra a las asignaturas del plan de estudios. De estos participantes, 68 son mujeres y 34 son hombres.

La selección fue por conveniencia. Primero, se consideraron todos aquellos que hubieran contestado el instrumento de retroalimentación en la plataforma de Coursera. Posteriormente, se seleccionaron solo los instrumentos que, en su respuesta, tuvieran relación con algún tipo de beneficio por haber participado en los MOOC. En la tabla 1, se muestra la distribución de los participantes.

Tabla 1 - Distribución y depuración de participantes

\begin{tabular}{|c|c|c|}
\hline $\begin{array}{c}\text { Alumnos que participaron en } \\
\text { algún curso MOOC }\end{array}$ & $\begin{array}{c}\text { Alumnos participantes } \\
\text { en la evaluación de } \\
\text { cursos MOOC }\end{array}$ & $\begin{array}{c}\text { Alumnos que contestaron } \\
\text { algún beneficio de haber } \\
\text { cursado un MOOC }\end{array}$ \\
\hline 4,700 & 272 & 102 \\
\hline
\end{tabular}

Fuente: Elaboración propia.

\section{Proceso y análisis}

A través de la plataforma de Coursera, se obtuvieron las 272 encuestas contestadas por los estudiantes, mismas que fueron revisadas manualmente para seleccionar aquellas que hicieran referencia a los beneficios de cursar un MOOC. Finalmente, después de este proceso, se seleccionaron las 102 encuestas que cumplían con dicho criterio, las cuales se consideraron como comentarios libres (CHARMAZ, 2006) y se utilizaron para llevar a cabo el análisis del presente estudio.

El análisis se desarrolló con el apoyo de tablas de contingencia en formato electrónico. Este fue inductivo (SALDAÑA, 2015) y consistió en una lectura de la totalidad de las respuestas, para identificar primeramente códigos y después la definición de categorías. Esto permitió la primera versión del libro de códigos. Con la intención de asegurar la calidad del proceso de análisis, a partir de la claridad, pertinencia y estructura (MILES; HUBERMAN, 1994), se realizó una validación por tres expertos nacionales y dos internacionales, quienes propusieron la fusión de dos categorías y la eliminación de cinco códigos para ser integrados en otros existentes, así como ajustes de redacción de 12 códigos. De esta manera, el libro de códigos quedó integrado de acuerdo con la tabla 2.

Tabla 2 - Integración del libro de códigos

\begin{tabular}{|c|l|}
\hline \multicolumn{1}{|c|}{ Categorías } & \multicolumn{1}{c|}{ Códigos } \\
\hline \multirow{4}{*}{ Desarrollo académico } & Confirmar lo aprendido en otras clases. \\
\cline { 2 - 2 } & Experimentar otras perspectivas del aprendizaje. \\
\cline { 2 - 2 } & Trabajar de forma autodidacta. \\
\cline { 2 - 2 } & Mejorar el proceso y experiencia de aprendizaje \\
\hline
\end{tabular}

Práxis Educativa, Ponta Grossa, v. 16, e2118097, p. 1-24, 2021 Disponível em: <https://revistas2.uepg.br/index.php/praxiseducativa $>$ 
Beneficios de los MOOC en estudiantes universitarios durante la emergencia académica por la covid-19

\begin{tabular}{|c|c|}
\hline Categorías & Códigos \\
\hline \multirow{4}{*}{ Desarrollo profesional } & Complementar la formación profesional. \\
\hline & Desarrollar aprendizajes de contenido propios de la carrera. \\
\hline & Mejorar la preparación para la incorporación laboral. \\
\hline & Desarrollar competencias digitales. \\
\hline \multirow{6}{*}{$\begin{array}{l}\text { Desarrollo personal y } \\
\text { motivación }\end{array}$} & Motivación para ampliar los aprendizajes. \\
\hline & Motivación para seguir estudiando la carrera elegida. \\
\hline & Motivación para seguir aprendiendo por medio de las tecnologías digitales \\
\hline & Motivación para ser mejor estudiante \\
\hline & Descubrir habilidades no identificadas. \\
\hline & Creer más en uno mismo. \\
\hline \multirow{3}{*}{ Flexibilidad } & Libertad de horarios. \\
\hline & Disponibilidad de contenidos. \\
\hline & Aprender a ritmo propio. \\
\hline \multirow{4}{*}{ Internacionalización } & Aprender en contextos externos a la universidad. \\
\hline & Mejorar el manejo de idioma extranjero. \\
\hline & Acceso a contenidos de universidades extranjeras. \\
\hline & Acceso a contenidos actualizados. \\
\hline \multirow{3}{*}{$\begin{array}{c}\text { Continuidad académica } \\
\text { (durante la emergencia académica } \\
\text { por la covid-19) }\end{array}$} & Complementar carga académica. \\
\hline & Evitar retraso en trayectoria académica. \\
\hline & Ampliación de la oferta formativa. \\
\hline
\end{tabular}

Fuente: Elaboración propia.

La tabla 3 presenta el ejemplo de uno de los códigos que integran el libro.

Tabla 3 - Ejemplo del código "Disponibilidad de contenidos"

\begin{tabular}{|c|l|}
\hline $\begin{array}{c}\text { Categoría } \\
\text { categoría }\end{array}$ & Flexibilidad \\
\hline Código & $\begin{array}{l}\text { Refiere a la libertad, en cuanto a márgenes de tiempo, que tienen los estudiantes para } \\
\text { participar en el curso, ya sea para alguna actividad en particular o la totalidad el curso. }\end{array}$ \\
\hline Definición del código & $\begin{array}{l}\text { Disponibilidad de contenidos. } \\
\text { Los contenidos del curso (de cualquier tipo), están en el curso/ plataforma cuando el alumno } \\
\text { los necesita, ya sea para una actividad en particular o siempre. }\end{array}$ \\
\hline Regla de codificación & $\begin{array}{l}\text { Codificar cuando se declare como beneficio lo relacionado a la disponibilidad de contenidos } \\
\text { en el curso/plataforma, ya sea en horario o temporalidad. }\end{array}$ \\
\hline Ejemplo de código & $\begin{array}{l}\text { "Me ha gustado mucho: es muy flexible con los horarios y las explicaciones siempre están } \\
\text { para verlas cuantas veces se necesite. Eso lo veo como un gran beneficio" (F-8-96). }\end{array}$ \\
\hline
\end{tabular}

Fuente: Elaboración propia.

Con la versión validada del libro de códigos y con la intención de asegurar la calidad del análisis y resultados (GLASER; STRAUSS, 1967), se realizó una nueva lectura de los comentarios atendiendo las reglas de codificación establecidas, lo que permitió la obtención de los resultados que a continuación se presentan.

\section{Resultados}

Se presentan de acuerdo con las seis categorías originadas a partir del análisis de contenido.

\section{Categoría 1: Desarrollo académico}

Esta categoría engloba cuatro códigos que permiten dar claridad sobre el desarrollo académico, entendido como un beneficio de los MOOC. Esto, desde la óptica de los estudiantes. Dicha categoría

Práxis Educativa, Ponta Grossa, v. 16, e2118097, p. 1-24, 2021 Disponível em: <https://revistas2.uepg.br/index.php/praxiseducativa> 
incluye la confirmación de lo aprendido en otras clases, la experimentación de otras perspectivas de aprendizaje, el trabajo de forma autodidacta y la mejora del proceso de aprendizaje.

De acuerdo con los comentarios de los alumnos, se identificó que cursar el MOOC les permitió confirmar lo aprendido en otras clases, debido a que identificaron contenidos que ya habían trabajado en otras asignaturas durante su formación universitaria: "Creo que los aprendizajes del MOOC me sirvieron para confirmar lo que aprendí en mis clases, y me hizo recordar" (DA-6-87). De igual forma, manifestaron que experimentaron otras perspectivas de aprendizaje; es decir, experiencias que no habían experimentado antes y a las cuales no estaban acostumbrados: "Muy práctico e interesante: abre perspectivas del aprendizaje a las cuales no estamos acostumbrados" (DA-2-89). Trabajar de forma autodidacta también fue un beneficio que los estudiantes identificaron, pues las experiencias implicaron que adecuaran sus propios esquemas de organización para aprender, que fuera necesario poner empeño, así como tener interés por aprender: "En algún libro leí que el mejor estudio es el autodidacta, y estoy completamente de acuerdo, pues de esta manera se nota el interés y empeño por lo que quieres aprender" (DA-3-74).

Como último elemento de la categoría, se identificó que los alumnos manifestaron una mejora en sus procesos y experiencias de aprendizaje; es decir, que en este tipo de cursos se visualiza una buena forma de aprender, la cual amplía las posibilidades de lograr los aprendizajes: "Para engrandecer el aprendizaje y las formas de aprender" (DA-4-27).

Los resultados de esta categoría coinciden con el estudio de Cripps (2014), quien identificó en estudiantes universitarios japoneses los beneficios de cursos MOOC, en particular con el desarrollo académico. De manera más particular, otros estudios como los de Loizzo y Ertmer (2016) se centraron entrevistar a adultos de diferentes nacionalidades y obtuvieron como resultado que experimentar otras perspectivas del aprendizaje es un elemento relevante de los MOOC. Por su parte, la investigación documental de literatura relacionada con los MOOC en educación superior, realizada por Al-Rahmi, Aldraiweesh, Yahaya, Bin Kamin y Zeki (2019) arrojó que los MOOC contribuyen a mejorar el proceso de aprendizaje, tal como se identificó en el presente estudio. Con este último punto, también en las narrativas de autores como Siemens (2012), Gómez (2014), Mazoué (2013), Stracke, Downes, Conole, Burgos y Nascimbeni (2019) y Frías, Arquero y Del Barrio-García (2020), se encontró coincidencia.

\section{Categoria 2: Desarrollo profesional}

Esta categoría incluye cuatro códigos que se asocian a beneficios en el ámbito de la formación profesional de los estudiantes. Incluye la posibilidad de complementar la formación profesional, el aprendizaje de contenidos propios de la carrera, mejorar la preparación para la incorporación laboral y el desarrollo de competencias digitales.

Los alumnos consideran que la experiencia de cursar un MOOC les ayudó a complementar su formación profesional, debido a que se trabajaron contenidos relacionados con lo que actualmente estaban cursando en sus clases: "Me parece que es un muy buen complemento para cualquier profesionista, pues los contenidos se apoyan en lo que estamos estudiando" (DP-1-59). Asimismo, desarrollaron el aprendizaje de contenidos propios de la carrera. En este sentido, los MOOC favorecen no solo contenidos genéricos, sino que también pueden favorecer aprendizajes disciplinares y profesionales: "Un curso que me ha servido en mi carrera, para aprender más a profundidad y también nuevos temas sobre mi licenciatura" (DP -5-26).

Práxis Educativa, Ponta Grossa, v. 16, e2118097, p. 1-24, 2021 
Beneficios de los MOOC en estudiantes universitarios durante la emergencia académica por la covid-19

También se identificó como beneficio la mejora en la preparación para la incorporación laboral, lo cual se logró a partir de contenidos propios de la disciplina o los relacionados con la inserción laboral, como lo son las competencias blandas. Este hallazgo puede estar relacionado con los tipos de MOOC que se ofertaron, los cuales fueron de contenidos disciplinares y contenidos genéricos como las habilidades blandas, las cuales no son específicas de una carrera: "Muy buen curso: te sirve no solo para mejorar lo relacionado a aspectos que nos encontremos en el ámbito laboral, sino también en el aspecto personal" (DP-6-75)

Finalmente, se indicó que la experiencia de cursar un MOOC permitió el desarrollo de competencias digitales. En particular, para el aprendizaje, pues los escenarios que ofrecieron los distintos cursos implicaron un aprendizaje con base en las tecnologías, en las que, conforme se avanzó en el proceso formativo, se obtuvo más confianza: "Creo que aprender a trabajar en este tipo de espacios virtuales fue muy grato y me aportó mucho, pues antes no me animaba a trabajar en línea" (DP-4-96).

Al comparar los resultados de esta categoría con otros estudios, se encontró una coincidencia. Un ejemplo de esto es el estudio de Loizzo y Ertmer (2016), quienes, como se mencionó anteriormente, entrevistaron a adultos de diferentes nacionalidades que participaron en un MOOC y concluyeron que esta modalidad permite desarrollar competencias digitales, pues el método de trabajo de esta modalidad requiere hacer uso de herramientas digitales para desarrollar el aprendizaje. Con este punto también concuerdan los trabajos de Siemens (2012) y Castaño y Cabero (2013).

Asimismo, autores como Díaz, Baena y Baena (2017) y Gómez (2014) establecen que los MOOC contribuyen al logro del desarrollo profesional al brindar opciones para complementar la formación profesional (DÍAZ; BAENA; BAENA, 2017; GÓMEZ, 2014). Por su parte, Cripps (2014), de acuerdo con los resultados de su estudio, indica que este tipo de experiencia mejora la preparación para la incorporación laboral.

\section{Categoría 3: Desarrollo personal y motivación}

La presente categoría contempla seis códigos que se asocian a beneficios relacionados con el desarrollo personal y motivación de los alumnos. Se integra por: motivación para ampliar los aprendizajes, motivación para seguir estudiando la carrera elegida, motivación para seguir aprendiendo por medio de las tecnologías digitales y motivación para ser mejor estudiante; además, por descubrir habilidades no identificadas y creer más en uno mismo.

Los participantes expresaron que cursar un MOOC contribuyó a que desarrollaran motivación para ampliar los aprendizajes: "Me encantó el curso y estoy decidida a aprender más. Terminé hasta emocionándome y queriéndolos más con cada clase” (DPM-5-47). Asimismo, los ayudó a desarrollar motivación para seguir estudiando la carrera elegida, pues sirvió para comprobar que están en la carrera correcta: "Me ayudó a querer y confirmar la carrera que estudio" (DPM-4-69). De la misma manera, comentaron que desarrollaron motivación para seguir aprendiendo por medio de las tecnologías digitales, debido a que fue su primera experiencia en esta modalidad y se sintieron satisfechos: "Nunca había cursado algo así, pero desde ahora seguiré con este tipo de cursos, aunque no me den créditos ni constancias" (DPM-4-569).

La motivación para ser mejor estudiante también fue un elemento que los participantes indicaron. Esto, gracias a la forma de trabajo y del proceso de aprendizaje de los MOOC: "La forma

Práxis Educativa, Ponta Grossa, v. 16, e2118097, p. 1-24, 2021 Disponível em: <https://revistas2.uepg.br/index.php/praxiseducativa> 
de aprender en el curso me motivó a mejorar como estudiante y así quiero seguir” (DPM-110). Al igual, descubrir habilidades no identificadas, tales como darse cuenta de que inconscientemente ya contaban con conocimientos: "Aprendí muchas cosas que no sabía que ya sabía; incluso, me sorprendî" (DPM-5-85)

Como último elemento de esta categoría, los participantes expresaron que cursar un MOOC los ayudó a creer más en sí mismos: "Era lo que necesitaba escolarmente y socialmente para crecer como estudiante y alcanzar mayores cosas que creía que no podía” (DPM-7-26).

Estos resultados concuerdan con otros estudios, como el aplicado por Mellati y Khademi (2018) a estudiantes universitarios iraníes, donde se identificó que la motivación es un aspecto que los MOOC contribuyen a desarrollar en los participantes. Lo mismo sucedió con Alhazzani (2020), quien descubrió, gracias a su estudio realizado con los docentes de una universidad de Arabia Saudita, que la motivación juega un papel relevante al momento de cursar un MOOC. Este aspecto también ha sido estudiado por diversos autores como Littlejohn, Hood, Milligan, and Mustain (2016) y Yough, Merzdorf, Fedesco y Cho (2017), por mencionar algunos.

\section{Categoría 4: Flexibilidad}

La cuarta categoría está integrada por tres códigos que se relacionan con los beneficios proporcionados por los MOOC en cuanto a flexibilidad. Incluye la libertad de horarios, disponibilidad de contenidos y aprender a ritmo propio.

Uno de los elementos expresados por los estudiantes refirió a la libertad de horarios con los que se puede trabajar, lo que les permite lograr una mejor organización: "Me ha gustado mucho. Es muy flexible con los horarios y eso me ayudó a organizarme mejor" (F-1-89). También se mencionó la disponibilidad de contenidos, ya que se puede acceder a estos en cualquier momento, lo cual resulta útil: "Me ha gustado mucho, porque las explicaciones siempre están para verlas cuantas veces se necesite. Eso lo veo como un gran beneficio" (F-8-96). Al igual, se comentó el beneficio de poder aprender a ritmo propio, ya que esto les otorga libertad en su proceso de aprendizaje: "La libertad de hacer las actividades cuando uno quiere y puede me gustó y ayudó mucho para aprender y poder cumplir con el curso" (F-8-36).

Estos puntos concuerdan con diversos estudios que expresan la flexibilidad como una de las ventajas de los MOOC (ALHAZZANI, 2020; MELLATI; KHADEMI, 2018). De manera particular, en el estudio de Alhazzani (2020), se identificó la libertad de horarios como un beneficio. Por otro lado, el estudio de Mellati y Khademi (2018), en el que participaron los estudiantes de universidad en Irán, se reconoció la disponibilidad de contenidos; mientras que Cripps (2014) identificó, en el contexto de estudiantes japoneses de una universidad, que aprender a ritmo propio era un beneficio considerado por los alumnos.

\section{Categoría 5: Internacionalización}

Esta categoría engloba un total de cuatro códigos que reúnen información sobre la internacionalización, entendida como un beneficio de los MOOC. La presente categoría incluye los siguientes elementos: aprender en contextos externos a la universidad, mejorar el manejo de un idioma extranjero, acceso a contenido de universidades extranjeras y acceso a contenidos actualizados.

Práxis Educativa, Ponta Grossa, v. 16, e2118097, p. 1-24, 2021 
Los estudiantes expresaron que su participación en el MOOC les sirvió para aprender en contextos externos a su propia universidad, pues tuvieron la oportunidad de interactuar con otro tipo de estudiantes, así como probar otras estrategias de trabajo: "Me ayudó a ver cómo aprenden y cómo trabajamos con otros estudiantes de distintas partes del mundo" (I-8-2).

Otro elemento mencionado fue la mejora en el manejo de un idioma extranjero, pues los recursos y materiales de los cursos estaban en otro idioma y esto permitió practicarlo: "Aunque el inglés no es mi lengua materna, me pareció que los contenidos y materiales que hay ayudan a mejorar el idioma" (I-56-75). Asimismo, se identificó como un beneficio el acceso a contenido de universidades extranjeras. Esto, por el hecho de que existen cursos diseñados por instituciones de educación superior internacionales que son impartidos por sus propios académicos: "Excelente la forma de abordar cada tema: son presentados por un experto distinto de otras universidades del mundo, lo que deja un gran aprendizaje y experiencia” (I-56-85).

Como último elemento dentro de esta categoría se encuentra el acceso a contenidos actualizados, ya que se consideró que estos eran útiles y avanzados: "No cabe duda de que la información da poder. Aquí se tuvieron conocimientos de primer nivel que me servirán mucho y los tendré antes que otros compañeros que no tomaron el curso" (I-45-8).

Al revisar los resultados, se puede notar que estos están de acuerdo con lo establecido por otros autores. Ejemplo de ello es el estudio efectuado por Rieber (2016), en el que participaron estudiantes y profesionales de educación superior, así como otros miembros de universidades. En dicho estudio se obtuvo que la internacionalización lograda por los MOOC es un elemento que los participantes mencionaron como positivo y de provecho para su desarrollo. De manera más específica, el estudio de Loizzo y Ertmer (2016) ya mencionado anteriormente, así como el informe elaborado por Durall, Gros, Maina, Johnson y Adams (2012), especifican que aprender en contextos externos a la universidad es un elemento beneficioso aportado por los MOOC. Asimismo, Cripps (2014) menciona la mejora del manejo de un idioma extranjero y el acceso a contenido de universidades extranjeras. Este último también revisado por Zambrano (2017).

En cuanto al acceso a contenidos actualizados, este elemento también ha sido mencionado encontrado como beneficio de acuerdo a los trabajos de diversos autores (CRIPPS, 2014; FRÍAS; ARQUERO; DEL BARRIO-GARCÍA, 2020; AL-RAHMI; ALDRAIWEESH; YAHAYA; BIN; ZEKI, 2019; CABERO; LLORENTE; VÁZQUEZ, 2014).

\section{Categoría 6: Continuidad académica (durante la emergencia académica por la covid-19)}

La presente y última categoría del estudio contempla tres códigos que se asocian a beneficios proporcionados por los MOOC y que tienen relación con la continuidad académica. En especial, con la situación actual durante la emergencia académica por la covid-19. Dichos elementos son: complementar carga académica, evitar retraso en trayectoria académica y ampliación de la oferta educativa.

Como ya se mencionó, el primer elemento refiere a que uno de los beneficios consiste en que permite complementar la carga académica, pues existió la opción de obtener créditos en este tipo de cursos: "Fue y será de gran ayuda para este semestre complicado por la pandemia, porque cursé más créditos de los que esperaba, ya que no todos los cursos de la facultad estaban en línea" (CA-56-85). También se expresó la ventaja de evitar retraso en la trayectoria académica, gracias a que permitió

Práxis Educativa, Ponta Grossa, v. 16, e2118097, p. 1-24, 2021 Disponível em: <https://revistas2.uepg.br/index.php/praxiseducativa> 
cumplir con requisitos que son necesarios: “iQué bueno que pude estar en el curso! Me ayudó a no tener que cursar un semestre más solo por una materia y así poder graduarme” (CA-8-91).

El último elemento mencionado en esta categoría y en el estudio en general fue la ampliación de la oferta formativa, porque permitió tener la oportunidad de más variedad de temas y materias para cursar: "Me ayudó a tener más variedad en mis materias, porque con este problema de llevar clases desde casa, no había temas nuevos y materias buenas en línea. Creo que esta sí me gustó más que otras" (CA-8-32).

Relativo a esta última categoría y los elementos que la conforman, los estudios de algunos de los autores mencionados con anterioridad coinciden con nuestros resultados. Por una parte, Alhazzani (2020) considera que los MOOC son una alternativa viable para contribuir a la continuidad académica, al aplicarlos como un complemento educativo en la educación a distancia, especialmente en la actualidad, debido a la contingencia sanitaria por la covid-19. En particular, uno de los estudios más recientes sobre el uso de MOOC en la contingencia es el de Purkayastha y Sinha (2021), quienes, por medio de su investigación en la Universidad Assam, de la India, concluyeron que este tipo de cursos demostró ser una opción formativa adecuada y aceptada por los estudiantes durante la pandemia. De la misma manera, Alhazanni (2020) y Conole (2015) establecen que cursar un MOOC contribuye a evitar un retraso en trayectoria académica, tanto por sus características como por los beneficios que estos aportan. Por otra parte, autores como Loizzo y Ertmer (2016), Al-Rahmi, Aldraiweesh, Yahaya, Bin, y Zeki (2019) y Alhazzani (2020) mencionan que pueden funcionar como una alternativa para ampliar la oferta formativa de los estudiantes.

\section{Conclusiones}

El trabajo de investigación presentado en este artículo se propuso identificar los beneficios de los MOOC en la formación de universitarios, durante un contexto de emergencia académica derivado de la covid-19. Los resultados permiten respaldar las siguientes conclusiones: existen beneficios, desde la óptica de estudiantes, y estos se integran en un conjunto de seis categorías: desarrollo académico, desarrollo profesional, desarrollo personal y motivación, flexibilidad, internacionalización y continuidad académica. De igual forma, en particular, se identifica un grupo de beneficios específicos para el escenario de contingencia, como la ampliación de la oferta de contenidos para tener más opciones de formación, permitiendo a los alumnos complementar su carga académica en la modalidad en línea y con ello evitar, en algunos casos, retrasos en su trayectoria escolar.

La evidencia sobre el uso de los MOOC en educación superior en México aún es limitada. Seguramente, debido a la contingencia académica, así como bajo los principios de flexibilidad y apertura curricular, un gran número de instituciones utilizaron este tipo de estrategias y en un futuro podrán valorar su incorporación en escenarios regulares tal como ha pasado en otros países. Esto implicará, principalmente, confiar en estos cursos ya sea de producción propia o externa. Sobre todo, si se quiere pasar de un nivel básico al usarlos como complemento a la formación, hasta la sustitución de cursos o contenidos de un plan de estudios formal.

El presente estudio da cuenta de una institución que, en tiempos de contingencia, asumió el reto y compromiso de permitir que sus estudiantes participaran en este tipo de cursos, y que a su vez tuvieran equivalencia con algunos de los créditos optativos que se ofrecen en el currículum formal. La

Práxis Educativa, Ponta Grossa, v. 16, e2118097, p. 1-24, 2021 
evidencia indica que dicha decisión fue acertada, ya que los estudiantes manifestaron un conjunto de beneficios asociados a su participación en esta estrategia.

La tendencia hacia la educación hibrida que se ha documentado en los últimos tiempos, y más ahora con la experiencia de la pandemia (HU; SPIRO, 2021; BABATUNDE; SOYKAN, 2020), pone de manifiesto la importancia de repensar algunos de los modelos pedagógicos (ESTELLES;FISCHMAN, 2020) y de buscar nuevas estrategias de enseñanza y aprendizaje apoyadas en la tecnología, en donde los MOOC se posicionan como una opción viable para complementar la formación educativa en procesos de emergencia y en la llamada nueva normalidad.

En cuanto a los alcances, limitaciones e importancia de la investigación, se pueden señalar como alcances que, a partir de los resultados, se aporta información sobre los beneficios de estudiar un MOOC; en particular, durante un proceso de contingencia. Sobre limitaciones, las conclusiones aquí vertidas no son definitivas, pues se basan en un caso o experiencia institucional, pero sí ofrecen información útil sobre el potencial de los MOOC en la formación de universitarios, tanto en esta contingencia como en futuras. El estudio toma valor, pues brinda un panorama sobre un escenario de contingencia, en donde los estudiantes se encontraron cursando asignaturas en línea y del mismo modo cursaron los MOOC. Como se puede observar en los resultados, esto tiene sus propias particularidades. Los hallazgos pueden ser de interés para investigadores, instituciones y tomadores de decisiones en materia de formación en línea.

Para finalizar, un elemento importante a indagar que no fue objeto de este trabajo, pero se suma a los posibles estudios próximos, es la tasa de finalización, ya que, en la experiencia de la universidad estudiada, la tasa de finalización fue de alrededor del $50 \%$, mientras que la literatura especializada indica que esta no pasa del 20\% (ALARCÓN, 2019; RIEBER, 2016; HOLLANDS; TIRTHALI, 2014). ¿Qué fue lo que generó esta alta tasa de finalización? ¿ fue la gratuidad del curso, así como del certificado o constancia? ¿el otorgamiento de créditos a su carga académica? ¿la no penalización en caso no de no finalizar el MOOC? ¿la necesidad de sumar créditos a su formación para no atrasarse por la falta de cursos en línea de la institución? Aclaramos que todas estas preguntas fueron condiciones que se establecieron en la estrategia de la institución estudiada en un contexto de contingencia académica, por lo que también se puede sumar la pregunta: ¿el contexto de contingencia académica se relaciona positivamente con la tasa de finalización?

\section{Referencias}

AKURATIYA, D.; MEDDAGE, D. Students' perception of online learning during COVID-19 pandemic: a survey study of IT students. International Journal of Research and Innovation in Social Science, v. 4, n. 9, p. 755-758, sep. 2020.

ALARCÓN, J. La trampa de la formación online sin fecha de fin. Campus MVP, mar. 2019. Recuperado el 15 de marzo de 2021 de: https://www.campusmvp.es/recursos/post/la-trampade-la-formacion-online-sin-fecha-de-fin.aspx. Accesado en: 23 ago. 2021.

ALBELBISI, N. A. Development and validation of the MOOC success scale (MOOC-SS). Education and Information Technologies, v. 25, n. 5, p. 4535-4555, abr. 2020 . DOI: https://doi.org/10.1007/s10639-020-10186-4 
ALHAZZANI, N. MOOC's impact on higher education. Social Sciences \& Humanities Open, v. 2, n. 1, p. 1-6. 2020. DOI: https://doi.org/10.1016/j.ssaho.2020.100030

ALLEN, E.; SEAMAN, J. Online report card: Tracking online education in the United States, 2016. Recuperado el 15 de marzo de 2021 de: https://onlinelearningsurvey.com/reports/onlinereportcard.pdf. Accesado en: 23 ago. 2021.

AL-RAHMI, W.; ALDRAIWEESH, A.; YAHAYA, N.; BIN, Y.; ZEKI, A. Massive Open Online Courses (MOOCs): Data on higher education. Data in brief, v. 22, p. 118-125, 2019. DOI: https://doi.org/10.1016/i.dib.2018.11.139

AMERICA LEARNING MEDIA. Debate MOOC: análisis e impacto de los cursos masivos abiertos en línea. Diciembre 2012. Recuperado el 15 de marzo de 2021 de: http://www.americalearningmedia.com/edicion-017/197-tendencias/2375-debate-mooc. Accesado en: 23 ago. 2021.

BABATUNDE, O.; SOYKAN, E. Covid-19 pandemic and online learning: the challenges and opportunities. Interactive Learning Environments, may. 2020. DOI: https://doi.org/10.1080/10494820.2020.1813180

BORDEN, J. MOOCs Are dead - Long live the MOOC. Wired, 2014. Recuperado el 15 de marzo de 2021 de: http://www.wired.com/2014/08/moocs-are-dead-long-live-the-mooc/. Accesado en: 23 ago. 2021.

BOZKURT, A.; KEEFER, J. Participatory learning culture and community formation in connectivist MOOCs. Interactive Learning Environments, v. 26, n. 6, p. 776-788, 2017. DOI: https://doi.org/10.1080/10494820.2017.1412988

BROWN, G. T. L. Schooling Beyond COVID-19: An unevenly distributed, future. Frontiers in Education, v. 5, n. 82, may. 2020. DOI: https://doi.org/10.3389/feduc.2020.00082

BRYSON, D. A timeline of the coronavirus pandemic. The New York Times. 2021. Recuperado en: https://www.nytimes.com/article/coronavirus-timeline.html. Accesado en: 23 ago. 2021.

BURDE, D.; KAPIT, A.; WAHL, R. L.; GUVEN, O.; SKARPETEIG, M. I. Education in emergencies: a review of theory and research. Review of Educational Research, v. 87, n. 3, p. 619658, nov. 2017. DOI: https://doi.org/10.3102/0034654316671594

CABERO, J.; LLORENTE, M.; VÁZQUEZ, A. Las tipologías de MOOC: su diseño e implicaciones educativas. Profesorado. Revista de Currículum y Formación de Profesorado, v. 18, n. 1, p. 1326, 2014.

CALISIR, F.; GUMUSSOY, C. A.; BAYRAKTAROGLU, A. E.; KARAALI, D. Predicting the intention to use a web-based learning system: perceived content quality, anxiety, perceived system quality, image, and the technology acceptance model. Human Factors and Ergonomics in Manufacturing and Service Industries, v. 24, n. 5, p. 515-531, 2014. DOI: https://doi.org/10.1002/hfm.20548

CARMONA, C. B. H.; FLORES, E. Y. E. Rezago educativo en escuelas públicas de Acapulco, Guerrero, ante la pandemia de covid-19. In: BRAMBILA, B.; LAY, I. (coord.). Propuestas de 
inclusión, educación y gestión cultural de jóvenes investigadores. México: Universidad de Guadalajara, 2021. p. 167-182.

CASTAÑO, C.; CABERO, J. Enseñar y aprender en entornos m-learning. Madrid: Síntesis, 2013.

CHARMAZ, K. Constructing grounded theory. A practical guide through qualitative analysis. California: Sage, 2006.

CHRISTAKIS, E.; CHRISTAKIS, N. A. Coronavirus: Closing the Schools Is Not the Only Option. The Atlantic. mar. 2020. Disponible en: https://www.theatlantic.com/ideas/archive/2020 /03/coronavirus-closingschools-not-only-option/608056/. Accesado en: 23 ago. 2021.

COMISIÓN ECONÓMICA PARA AMÉRICA LATINA Y EL CARIBE. Personas con discapacidad y sus derechos frente a la pandemia de COVID-19: que nadie se quede atrás. 2021. Recuperado el 20 de agosto de 2021 de: https://repositorio.cepal.org/bitstream/handle /11362/46600/1/S2000791_es.pdf. Accesado en: 23 ago. 2021.

CONOLE, G. Designing effective MOOCs. Educational Media International, v. 52, n. 4, p. 239252, 2015. DOI: https://doi.org/10.1080/09523987.2015.1125989

CRIPPS, T. It's my challenge: exploring the MOOC terrain. The 6th CLS international conference. Conference proceedings, p. 89-102, 2014. Recuperado el 15 de marzo de 2021 de: https://bit.ly/3zfVUt6. Accesado en: 23 ago. 2021.

DELONE, W. H.; MCLEAN, E. R. The DeLone and McLean model of information systems success: A ten-year update. Journal of Management Information Systems, v. 19, n. 4, p. 9-30, 2003. DOI: https://doi.org/10.1080/07421222.2003.11045748

DÍAZ, Y.; BAENA, M.; BAENA, G. MOOC en la educación: un acercamiento al estado de conocimiento en Iberoamérica, 2014-2017. Revista Iberoamericana para la Investigación y el Desarrollo Educativo, v. 8, n. 15, jul/dic. 2017.

DIRIBA, S.; KANNAN, R.; CHAI, I. Updated DeLone and McLean IS success model and commercial open source software (COSS) company success. Knowledge Management International $\begin{array}{lllll}\text { Conference } & \text { (KMICe) 2021, feb. } & 2021\end{array}$ http://www.kmice.cms.net.my/ProcKMICe/KMICe2021/pdf/CR136.pdf. Accesado en: 23 ago. 2021.

DOWNES, S. The rise of MOOCs. 2012. Recuperado el 15 de marzo de 2021 de: https://www.downes.ca/post/57911. Accesado en: 23 ago. 2021.

DRANE, C.; VERNON, L.; O'SHEA, S. The impact of 'learning at home' on the educational outcomes of vulnerable children in Australia during the COVID-19 pandemic. abr. 2020. Recuperado el 20 de agosto de 2021 de: https://www.ncsehe.edu.au/wpcontent/uploads/2020/04/NCSEHE_V2_Final_literaturereview-learningathome-covid19-

final_30042020.pdf. Accesado en: 23 ago. 2021.

DURALL, E.; GROS, B.; MAINA, M.; JOHNSON, L.; ADAMS, S. Perspectivas tecnológicas: educación superior en Iberoamérica 2012-2017. 2012. Recuperado el 15 de marzo de 2021 de: 
https://www.academia.edu/4603109/Perspectivas_Tecnol\%C3\%B3gicas_Educaci\%C3\%B3n_Supe rior_en_Iberoam\%C3\%A9rica_2012_2017. Accesado en: 23 ago. 2021.

DZIUBAN, C.; GRAHAM, C.; MOSKAL, P.; NORBERG, A.; SICILIA, N. Blended learning: the new normal and emerging technologies. International Journal of Educational Technology in Higher Education, v. 15, n. 3, p. 1-16, 2018. DOI: https://doi.org/10.1186/s41239-017-0087-5

ESTELLES, M.; FISCHMAN, G. Imaginando una educación para la ciudadanía global después del COVID-19. Praxis Educativa, v. 15, 1-14. 2020. https://doi.org/10.5212/PraxEduc.v.15.15566.051

FIRMIN, R.; SCHIORRING, E.; WHITMER, J.; WILLETT, T.; COLLINS, E. D.; SUJITPARAPITAYA, S. Case study: using MOOCs for conventional college coursework. Distance Education, v. 35, n. 2, p. 178-201, jun. 2014. DOI: https://doi.org/10.1080/01587919.2014.917707

FRÍAS, J.; ARQUERO, J.; DEL BARRIO-GARCÍA. Exploring how student motivation relates to acceptance and participation in MOOCs. Interactive Learning Environments, p. 1-17, 2020. DOI: https://doi.org/10.1080/10494820.2020.1799020

GLASER, B.; STRAUSS, A. The discovery of grounded research: strategies for qualitative research. New York: Aldine, 1967.

GÓMEZ, T. Los MOOCs y su papel en la educación universitaria. 2014. Recuperado el 15 de marzo de 2021 de: http://www.inaecu.com/55736/\#: :text=Beneficios\%20potenciales $\% 3 \mathrm{~A} \% 20$ mejorar $\% 201 \mathrm{a} \% 20$ presencia,fuera $\% 20 \mathrm{del}^{2} \% 20 \% \mathrm{C} 3 \% \mathrm{~A} 1 \mathrm{mbito} \% 20 \mathrm{de} \% 20 \mathrm{la}$. Accesado en: 23 ago. 2021.

GRAINGER, B. Introduction to MOOCs: avalanche, illusion or augmentation? 2013. Recuperado el 15 de marzo de 2021 de: http://unesdoc.unesco.org/images/0022/002238/223896e.pdf. Accesado en: 23 ago. 2021.

HODGES, C.; MOORE, S.; LOCKEE, B.; TRUST, T.; BOND, A. The difference between emergency remote teaching and online learning. Educase Review. Recuperado el 15 de marzo de 2021 de: https://er.educause.edu/articles/2020/3/the-difference-between-emergency-remoteteaching-and-online-learning. Accesado em: 23 ago. 2021.

HOLLANDS, F. M.; TIRTHALI, D. (2014). MOOCs: expectations and reality, Full Report. 2014. Recuperado el 15 de marzo de 2021 de: https://www.researchgate.net/publication/271841177_MOOCs_Expectations_and_reality.

Accesado en: 23 ago. 2021.

HU, Y.; SPIRO, R. J. Design for now, but with the future in mind: a "cognitive flexibility theory" perspective on online learning through the lens of MOOCs. Educational Technology Research and Development, v. 20, n. 1, p. 373-378, ene. 2021. DOI: https://doi.org/10.1007/s11423-020$\underline{09920-z}$

HUGHES, C. What lessons from the coronavirus pandemic will shape the future of education? mai. 2020. Recuperado el 20 de agosto de 2021 de: https://www.weforum.org/agenda/2020/05/covid19-lockdown-future-education/. Accesado en: 23 ago. 2021. 
Beneficios de los MOOC en estudiantes universitarios durante la emergencia académica por la covid-19

HUMAN RIGHTS WATCH. “Years Don't Wait for Them” increased inequalities in children's right to education due to the covid-19 pandemic. mai. 2021. https://www.hrw.org/sites/default/files/media_2021/05/global_covideducation0521_web.pdf.

Accesado en: 23 ago. 2021.

KOPP, M.; GRÖBLINGER, O.; ADAMS, S. Five common assumptions that prevent digital transformation at higher education institutions. INTED2019 Proceedings, p. 1448-1457, mar. 2019. DOI: https://doi.org/10.21125/inted.2019

LABORDE, T.; ORTEGA, A. 7 beneficios de los MOOC para las PyMEs. 2016. Recuperado el 15 de marzo de 2021 de: https://idbinvest.org/es/blog/7-beneficios-de-los-moocs-para-las-pymes. Accesado en: 23 ago. 2021.

LITTLEJOHN, A.; HOOD, N.; MILLIGAN, C.; MUSTAIN, P. Learning in MOOCs: Motivations and self-regulated learning in MOOCs. Internet and Higher Education, v. 29, p. 40-48, dic. 2016. DOI: https://doi.org/10.1016/j.iheduc.2015.12.003

LOIZZO, J.; ERTMER, P. MOOCocracy: the learning culture of massive open online courses. Educational Technology Research and Development, v. 64, n. 6, p. 1013-1032, abr. 2016. DOI: https://doi.org/10.1007/s11423-016-9444-7

MACKNESS, J.; WAITE, M.; ROBERTS, G.; LOVEGROVE, E. Learning in a small, task-oriented, connectivist MOOC: Pedagogical issues and implications for higher education. The International Review of Research in Open and Distributed Learning, v. 14, n. 4, p. 140-159, sep. 2013. DOI: https://doi.org/10.19173/irrodl.v14i4.1548

MAZOUÉ, J. The MOOC model: challenging traditional education. Educase Review. ene. 2013. Recuperado el 15 de marzo de 2021 de: https://er.educause.edu/articles/2013/1/the-mooc-modelchallenging-traditional-education. Accesado en: 23 ago. 2021.

MELLATI, M.; KHADEMI, M. MOOC-based educational program and interaction in distance education: long life mode of teaching. Interactive Learning Environments, v. 28, n. 8, p. 1-14, dic. 2018. DOI: https://doi.org/10.1080/10494820.2018.1553188

MERRIAM, S.; TISDELL, E. Qualitative research: a guide to design and implementation. California: John Wiley \& Sons, 2015.

MILES, M.; HUBERMAN, A. Qualitative data analysis: a sourcebook of new methods. California: Sage, 1994.

NAGASHIMA, T. What makes open education thrive? Examination of factors contributing the success of open education initiatives. The International Journal for Innovation and Quality in Learning (INNOQUAL), v. 3, p. 10-21, sep. 2014.

NÄSLUND-HADLEY, E.; BIEHL, M. El involucramiento de padres en la educación a distancia y la brecha de género en salud mental. oct. 2020. Recuperado el 20 de agosto de 2021 de: https://blogs.iadb.org/educacion/es/involucramientodepadres/. Accesado en: 23 ago. 2021.

Práxis Educativa, Ponta Grossa, v. 16, e2118097, p. 1-24, 2021 Disponível em: <https://revistas2.uepg.br/index.php/praxiseducativa> 
ONYEMA, E.; EUCHERIA, N.; OBAFEMI, F.; SEN, S.; ATONYE, F.; SHARMA, A.; ALSAYED, A. Impact of coronavirus pandemic on education. Journal of Education and Practice, v. 11, n. 13, p. 108-121, mayo, 2020. DOI: https://doi.org/10.7176/jep/11-13-12

ORGANIZACIÓN DE ESTADOS IBEROAMERICANOS PARA LA EDUCACIÓN, LA CIENCIA Y LA CULTURA (OEI). (2019). Observatorio Iberoamericano de la Ciencia, la Tecnología y la Sociedad, Informe de Coyuntura, No 6, 2019. Recuperado el 15 de marzo de 2021 de: http://octs-oei.org/coyuntura/coyuntura06.html. Accesado en: 23 ago. 2021.

PADILLA, B.; ROCHA, F.; RODRÍGUEZ, M. Razones para estudiar un curso en línea masivo y abierto (MOOC) de habilidades de estudio. 2017. Recuperado el 15 de marzo de 2021 de: http://ceur-ws.org/Vol-1836/6.pdf. Accesado en: 23 ago. 2021.

PARDO, H.; COBO, C. Expandir la universidad más allá de la enseñanza remota de emergencia Ideas hacia un modelo híbrido post-pandemia. Barcelona: Outliers School, 2020.

PURKAYASTHA, N.; SINHA, M. K. Unstoppable study with MOOCs during covid 19 pandemic: a study. Library Philosophy and Practice, p. 1-12, ene. 2021.

RIBEIRO, R. How university faculty embraced the remote learning shift. EdTech Magazine, apr. 2020. Recuperado el 15 de marzo de 2021 de: https://edtechmagazine.com/higher/article/2020/04/how-university-faculty-embraced-remotelearning-shift. Accesado en: 23 ago. 2021.

RIEBER, L. Participation patterns in a massive open online course (MOOC) about statistics. British Journal of Educational Technology, v. 48, n. 6, p. 1295-1304, ago. 2016. DOI: https://doi.org/10.1111/bjet.12504

RUIZ, C. (2015). El MOOC: ¿un modelo alternativo para la educación universitaria? Revista Apertura, v. 7, n. 2, oct. 2015.

RUSSO, K.; MAGNAN, M.; SOARES, R. A pandemia que amplia as desigualdades: a Covid-19 e o sistema educativo de Queben/Canadá. Praxis Educativa, v. 15, 1-28. 2020. https://doi.org/10.5212/PraxEduc.v.15.15915.073

SALDAÑA, J. The coding manual for qualitative researchers. California: Sage, 2015.

SIEMENS, G. MOOCs are really a platform. eLearnspace. 2012. Recuperado de: http://www.elearnspace.org/blog/2012/07/25/moocs-are-really-a-platform/. Accesado en: 23 ago. 2021.

STRACKE, C.; DOWNES, S.; CONOLE, G.; BURGOS, D.; NASCIMBENI, F. Are MOOCs open educational resources? A literature review on history, definitions and typologies of OER and MOOCs. Open Praxis, v. 11, n. 4, p. 331-341, dic. 2019. DOI: https://doi.org/10.5944/openpraxis.11.4.1010

UNESCO/IESALC. COVID-19 y educación superior: de los efectos inmediatos al día después. Análisis de impactos, respuestas políticas y recomendaciones. mai. 2020. Recuperado el 14 de abril de 2021 de: http://www.iesalc.unesco.org/wp-content/uploads/2020/05/COVID-19-ES-130520.pdf.

Accesado en: 23 ago. 2021. 
UNICEF. COVID-19 and school closures. UNICEF DATA. 2021. Recuperado el 20 de agosto de 2021 de: https:/ / data.unicef.org/resources/one-year-of-covid-19-and-school-closures/. Accesado en: 23 ago. 2021.

VALENTI, M. La pandemia de las desigualdades. oct. 2020. Recuperado el 20 de agosto de 2021 de: https://www.clacso.org/la-pandemia-de-las-desigualdades/. Accesado en: 23 ago. 2021.

YANG, M.; SHAO, Z.; LIU, Q.; LIU, C. Understanding the quality factors that influence the continuance intention of students toward participation in MOOCs. Educational Technology Research and Development, v. 65, p. 1195-1214, feb. 2017. DOI: https://doi.org/10.1007/s11423017-9513-6

YOUGH, M.; MERZDORF, H.; FEDESCO, H.; CHO, H. Flipping the classroom in teacher education: Implications for motivation and learning. Journal of Teacher Education, v. 70, n. 5, p. 1-13, nov. 2017.

ZAMBRANO, E. Algunos beneficios de los MOOCs: Cursos Online Masivos y Abiertos. Blog edx. nov. 2017. Recuperado el 15 de marzo de 2021 de: https://blog.edx.org/es/algunos-beneficios-delos-moocs-cursos-online-masivos-y-abiertos. Accesado en: 23 ago. 2021.

Recibido: 03/06/2021

Versión corregida recibida: 24/08/2021

Aceptado: 25/08/2021

Publicado online: $26 / 08 / 2021$ 